\title{
Fractional maximal commutator on Orlicz spaces for the Dunkl operator on the real line
}

\author{
Yagub Y. Mammadov * Fatma A. Muslumova · Zaman V. \\ Safarov
}

Received: 15.03.2020 / Revised: 30.09.2020 / Accepted: 07.11.2020

Abstract. On the real line, the Dunkl operators

$$
D_{\nu}(f)(x):=\frac{d f(x)}{d x}+(2 \nu+1) \frac{f(x)-f(-x)}{2 x}, x \in \mathbb{R}, \nu \geq-1 / 2
$$

are differential-difference operators associated with the reflection group $\mathbb{Z}_{2}$ on $\mathbb{R}$. In this paper, we find necessary and sufficient conditions for the boundedness of the fractional maximal operator $M_{\alpha, \nu}$ on Orlicz spaces $L_{\Phi}\left(\mathbb{R}, d m_{\nu}\right)$. As an application of this result we show that $b \in B M O\left(\mathbb{R}, d m_{\nu}\right)$ if and only if the fractional maximal commutator $M_{b, \alpha, \nu}$ is bounded on Orlicz spaces $L_{\Phi}\left(\mathbb{R}, d m_{\nu}\right)$.

Keywords. Fractional maximal operator; Orlicz space; Dunkl operator; commutator; $B M O$

Mathematics Subject Classification (2010): 42B20, 42B25, 42B35

\section{Introduction}

Norm inequalities for several classical operators of harmonic analysis have been widely studied in the context of Orlicz spaces. It is well known that many of such operators fail to have continuity properties when they act between certain Lebesgue spaces and, in some situations, the Orlicz spaces appear as adequate substitutes. For example, the Hardy-Littlewood maximal operator is bounded on $L_{p}$ for $1<p<\infty$, but not on $L_{1}$, but using Orlicz spaces, we can investigate the boundedness of the maximal operator near $p=1$, see [14] and [4] for more precise statements.

\footnotetext{
* Corresponding author

Y.Y. Mammadov

Nakhchivan Teacher-Training Institute, Azerbaijan

Department of Informatics, Nakhchivan State University, Azerbaijan

E-mail: yagubmammadov@yahoo.com

F.A. Muslumova

Department of Informatics, Nakhchivan State University, Azerbaijan

E-mail: fmuslumova@gmail.com

Z.V. Safarov

Azerbaijan State Oil and Industry University, Baku, Azerbaijan

Institute of Mathematics and Mechanics of NAS of Azerbaijan, Baku, Azerbaijan

E-mail: zsafarov@gmail.com
} 
Let $T$ be the classical singular integral operator, the commutator $[b, T]$ generated by $T$ and a suitable function $b$ is given by

$$
[b, T] f:=b T(f)-T(b f) .
$$

A well-known result due to Coifman, Rochberg and Weiss [3] (see also [12]) states that $b \in B M O\left(\mathbb{R}^{n}\right)$ if and only if the commutator $[b, T]$ is bounded on $L_{p}\left(\mathbb{R}^{n}\right)$ for $1<p<\infty$.

On the real line, the Dunkl operators $\Lambda_{\nu}$ are differential-difference operators introduced in 1989 by Dunkl [9]. For a real parameter $\nu \geq-1 / 2$, we consider the Dunkl operator, associated with the reflection group $\mathbb{Z}_{2}$ on $\mathbb{R}$ :

$$
D_{\nu}(f)(x):=\frac{d f(x)}{d x}+(2 \nu+1) \frac{f(x)-f(-x)}{2 x}, x \in \mathbb{R} .
$$

Note that $D_{-1 / 2}=d / d x$.

It is well known that maximal and fractional maximal operators play an important role in harmonic analysis (see [23]). Also the fractional maximal function and the fractional integral, associated with $D_{\nu}$ differential-difference Dunkl operators play an important role in Dunkl harmonic analysis, differentiation theory and PDE's. The harmonic analysis of the one-dimensional Dunkl operator and Dunkl transform was developed in $[6,7,16]$. The Dunkl operator and Dunkl transform considered here are the rank-one case of the general Dunkl theory, which is associated with a finite reflection group acting on a Euclidean space. The Dunkl theory provides a useful framework for the study of multivariable analytic structures and has gained considerable interest in various fields of mathematics and in physical applications (see, for example, [10]). The fractional maximal function, the fractional integral and related topics associated with the Dunkl differential-difference operator have been research areas for many mathematicians such as C. Abdelkefi and M. Sifi [1], V.S. Guliyev and Y.Y. Mammadov [6-8], Y.Y. Mammadov [15], L. Kamoun [13], M.A. Mourou [17], F. Soltani [21,22], K. Trimeche [24] and others. Moreover, the results on $L_{\Phi}\left(\mathbb{R}, d m_{\nu}\right)$-boundedness of maximal operators associated with $D_{\nu}$ were obtained in [8], where $d m_{\nu}(x):=\left(2^{\nu+1} \Gamma(\nu+1)\right)^{-1}|x|^{2 \nu+1} d x$.

In this paper, in the framework of the Dunkl analysis in the setting $\mathbb{R}$, we shall give a necessary and sufficient condition for the boundedness of fractional maximal operator $M_{\alpha, \nu}$ on Orlicz spaces $L_{\Phi}\left(\mathbb{R}, d m_{\nu}\right)$ and weak Orlicz spaces $W L_{\Phi}\left(\mathbb{R}, d m_{\nu}\right)$. Also, we study the boundedness of the fractional maximal commutator $M_{b, \alpha, \nu}$ and the commutator of the fractional maximal operator, $\left[b, M_{\alpha, \nu}\right]$, on Orlicz spaces $L_{\Phi}\left(\mathbb{R}, d m_{\nu}\right)$, when $b$ belongs to the space $B M O\left(\mathbb{R}, d m_{\nu}\right)$, by which some new characterizations of the space $B M O\left(\mathbb{R}, d m_{\nu}\right)$ are given.

By $A \lesssim B$ we mean that $A \leq C B$ with some positive constant $C$ independent of appropriate quantities. If $A \lesssim B$ and $B \lesssim A$, we write $A \approx B$ and say that $A$ and $B$ are equivalent.

\section{Preliminaries in the Dunkl setting on $\mathbb{R}$} by

Let $\nu>-1 / 2$ be a fixed number and $m_{\nu}$ be the weighted Lebesgue measure on $\mathbb{R}$, given

$$
d m_{\nu}(x):=\left(2^{\nu+1} \Gamma(\nu+1)\right)^{-1}|x|^{2 \nu+1} d x, \quad x \in \mathbb{R} .
$$

For any $x \in \mathbb{R}$ and $r>0$, let $B(x, r):=\{y \in \mathbb{R}:|y| \in] \max \{0,|x|-r\},|x|+r[\}$. Then $B(0, r)=]-r, r\left[\right.$ and $m_{\nu} B(0, r)=c_{\nu} r^{2 \nu+2}$, where $c_{\nu}:=\left[2^{\nu+1}(\nu+1) \Gamma(\nu+1)\right]^{-1}$. 
The fractional maximal operator $M_{\alpha, \nu}, 0 \leq \alpha<2 \nu+2$ associated by Dunkl operator on the real line is given by

$$
M_{\alpha, \nu} f(x):=\sup _{r>0}\left(m_{\nu} B(x, r)\right)^{-1+\frac{\alpha}{2 \nu+2}} \int_{B(x, r)}|f(y)| d m_{\nu}(y), \quad x \in \mathbb{R}
$$

and the fractional maximal commutator $M_{b, \alpha, \nu}, 0 \leq \alpha<2 \nu+2$ associated with Dunkl operator on the real line and with a locally integrable function $b \in L_{1}^{\text {loc }}\left(\mathbb{R}, d m_{\nu}\right)$ is defined by

$$
M_{b, \alpha, \nu} f(x):=\sup _{r>0}\left(m_{\nu} B(x, r)\right)^{-1+\frac{\alpha}{2 \nu+2}} \int_{B(x, r)}|b(x)-b(y)||f(y)| d m_{\nu}(y), \quad x \in \mathbb{R} .
$$

If $\alpha=0$, then $M_{\nu} \equiv M_{\nu, 0}$ is the maximal operator associated by Dunkl operator on the real line and $M_{b, \nu} \equiv M_{b, \nu, 0}$ is the maximal commutator operator associated by Dunkl operator on the real line.

On the other hand, similar to (1.1), we can define the (nonlinear) commutator of the fractional maximal operator $M_{\alpha, \nu}$ with a locally integrable function $b$ by

$$
\left[b, M_{\alpha, \nu}\right](f)(x) f=b(x) M_{\alpha, \nu}(f)(x)-M_{\alpha, \nu}(b f)(x) .
$$

For more details about the operators $M_{b, \nu}$ and $\left[b, M_{\nu}\right.$ ], we refer to [8] and references therein.

For a function $b$ defined on $\mathbb{R}$, we let, for any $x \in \mathbb{R}$,

$$
b^{-}(x):= \begin{cases}0, & \text { if } b(x) \geq 0 \\ |b(x)|, & \text { if } b(x)<0\end{cases}
$$

and $b^{+}(x):=|b(x)|-b^{-}(x)$. Obviously, for any $x \in \mathbb{R}, b^{+}(x)-b^{-}(x)=b(x)$.

The following relations between $\left[b, M_{\alpha, \nu}\right]$ and $M_{b, \alpha, \nu}$ are valid:

Let $b$ be any non-negative locally integrable function. Then

$$
\left|\left[b, M_{\alpha, \nu}\right] f(x)\right| \leq M_{b, \alpha, \nu}(f)(x), \quad x \in \mathbb{R}
$$

holds for all $f \in L_{1}^{\text {loc }}\left(\mathbb{R}, d m_{\nu}\right)$.

If $b$ is any locally integrable function on $\mathbb{R}$, then

$$
\left|\left[b, M_{\alpha, \nu}\right] f(x)\right| \leq M_{b, \alpha, \nu}(f)(x)+2 b^{-}(x) M_{\alpha, \nu} f(x), \quad x \in \mathbb{R}
$$

holds for all $f \in L_{1}^{\operatorname{loc}}\left(\mathbb{R}, d m_{\nu}\right)$ (see, for example, [2]).

\subsection{Orlicz spaces in the Dunkl setting on $\mathbb{R}$}

Recall that Orlicz space was first introduced by Orlicz in $[18,19]$ as a generalizations of Lebesgue spaces $L_{p}$. Since then this space has been one of important functional frames in the mathematical analysis, and especially in real and harmonic analysis. Orlicz space is also an appropriate substitute for $L_{1}$ space when the space $L_{1}$ does not work.

To introduce the notion of Orlicz spaces in the Dunkl setting on $\mathbb{R}$, we first recall the definition of Young functions.

Definition 2.1 A function $\Phi:[0, \infty) \rightarrow[0, \infty]$ is called a Young function if $\Phi$ is convex, left-continuous, $\lim _{r \rightarrow+0} \Phi(r)=\Phi(0)=0$ and $\lim _{r \rightarrow \infty} \Phi(r)=\infty$. 
From the convexity and $\Phi(0)=0$ it follows that any Young function is increasing. If there exists $s \in(0, \infty)$ such that $\Phi(s)=\infty$, then $\Phi(r)=\infty$ for $r \geq s$. The set of Young functions such that

$$
0<\Phi(r)<\infty \quad \text { for } \quad 0<r<\infty
$$

is denoted by $\mathcal{Y}$. If $\Phi \in \mathcal{Y}$, then $\Phi$ is absolutely continuous on every closed interval in $[0, \infty)$ and bijective from $[0, \infty)$ to itself.

For a Young function $\Phi$ and $0 \leq s \leq \infty$, let

$$
\Phi^{-1}(s):=\inf \{r \geq 0: \Phi(r)>s\} .
$$

If $\Phi \in \mathcal{Y}$, then $\Phi^{-1}$ is the usual inverse function of $\Phi$. It is well known that

$$
r \leq \Phi^{-1}(r) \widetilde{\Phi}^{-1}(r) \leq 2 r \quad \text { for any } r \geq 0,
$$

where $\widetilde{\Phi}(r)$ is defined by

$$
\widetilde{\Phi}(r):=\left\{\begin{array}{cc}
\sup \{r s-\Phi(s): s \in[0, \infty)\}, & r \in[0, \infty) \\
\infty, & r=\infty .
\end{array}\right.
$$

A Young function $\Phi$ is said to satisfy the $\Delta_{2}$-condition, denoted also as $\Phi \in \Delta_{2}$, if

$$
\Phi(2 r) \leq C \Phi(r), \quad r>0
$$

for some $C>1$. If $\Phi \in \Delta_{2}$, then $\Phi \in \mathcal{Y}$. A Young function $\Phi$ is said to satisfy the $\nabla_{2}$-condition, denoted also by $\Phi \in \nabla_{2}$, if

$$
\Phi(r) \leq \frac{1}{2 C} \Phi(C r), \quad r \geq 0
$$

for some $C>1$. In what follows, for any subset $E$ of $\mathbb{R}$, we use $\chi_{E}$ to denote its characteristic function.

Definition 2.2 (Orlicz Space). For a Young function $\Phi$, the set

$$
L_{\Phi}\left(\mathbb{R}, d m_{\nu}\right):=\left\{f \in L_{1}^{\mathrm{loc}}\left(\mathbb{R}, d m_{\nu}\right): \int_{\mathbb{R}} \Phi(k|f(x)|) d m_{\nu}(x)<\infty \text { for some } k>0\right\}
$$

is called the Orlicz space. If $\Phi(r):=r^{p}$ for all $r \in[0, \infty), 1 \leq p<\infty$, then $L_{\Phi}\left(\mathbb{R}, d m_{\nu}\right)=$ $L_{p}\left(\mathbb{R}, d m_{\nu}\right)$. If $\Phi(r):=0$ for all $r \in[0,1]$ and $\Phi(r):=\infty$ for all $r \in(1, \infty)$, then $L_{\Phi}\left(\mathbb{R}, d m_{\nu}\right)=L_{\infty}\left(\mathbb{R}, d m_{\nu}\right)$. The space $L_{\Phi}^{\mathrm{loc}}\left(\mathbb{R}, d m_{\nu}\right)$ is defined as the set of all functions $f$ such that $f \chi_{B} \in L_{\Phi}\left(\mathbb{R}, d m_{\nu}\right)$ for all balls $B \subset \mathbb{R}$.

$L_{\Phi}\left(\mathbb{R}, d m_{\nu}\right)$ is a Banach space with respect to the norm

$$
\|f\|_{L_{\Phi, \nu}}:=\inf \left\{\lambda>0: \int_{\mathbb{R}} \Phi\left(\frac{|f(x)|}{\lambda}\right) d m_{\nu}(x) \leq 1\right\} .
$$

For a measurable function $f$ on $\mathbb{R}$ and $t>0$, let

$$
m(f, t)_{\nu}:=m_{\nu}\{x \in \mathbb{R}:|f(x)|>t\} .
$$

Definition 2.3 The weak Orlicz space

$$
W L_{\Phi}\left(\mathbb{R}, d m_{\nu}\right):=\left\{f \in L_{1, \nu}^{\mathrm{loc}}(\mathbb{R}):\|f\|_{W L_{\Phi, \nu}}<\infty\right\}
$$

is defined by the norm

$$
\|f\|_{W L_{\Phi, \nu}}:=\inf \left\{\lambda>0: \sup _{t>0} \Phi(t) m\left(\frac{f}{\lambda}, t\right)_{\nu} \leq 1\right\} .
$$


We note that $\|f\|_{W L_{\Phi, \nu}} \leq\|f\|_{L_{\Phi, \nu}}$,

$$
\sup _{t>0} \Phi(t) m(f, t)_{\nu}=\sup _{t>0} t m\left(f, \Phi^{-1}(t)\right)_{\nu}=\sup _{t>0} t m(\Phi(|f|), t)_{\nu}
$$

and

$$
\int_{\mathbb{R}} \Phi\left(\frac{|f(x)|}{\|f\|_{L_{\Phi, \nu}}}\right) d m_{\nu}(x) \leq 1, \quad \sup _{t>0} \Phi(t) m\left(\frac{f}{\|f\|_{W L_{\Phi, \nu}}}, t\right)_{\nu} \leq 1 .
$$

The following analogue of the Hölder inequality is well known (see, for example, [20]).

Theorem 2.1 Let the functions $f$ and $g$ be measurable on $\mathbb{R}$. For a Young function $\Phi$ and its complementary function $\widetilde{\Phi}$, the following inequality is valid

$$
\int_{\mathbb{R}}|f(x) g(x)| d m_{\nu}(x) \leq 2\|f\|_{L_{\Phi, \nu}}\|g\|_{L_{\tilde{\Phi}, \nu}} .
$$

By elementary calculations we have the following property.

Lemma 2.1 Let $\Phi$ be a Young function and $B$ be a ball in $\mathbb{R}$. Then

$$
\left\|\chi_{B}\right\|_{L_{\Phi, \nu}}=\left\|\chi_{B}\right\|_{W L_{\Phi}\left(\mathbb{R}, d m_{\nu}\right)}=\frac{1}{\Phi^{-1}\left(\left(m_{\nu}(B)\right)^{-1}\right)} .
$$

By Theorem 2.1, Lemma 2.1 and (2.2) we obtain the following estimate.

Lemma 2.2 For a Young function $\Phi$ and for the ball $B$ the following inequality is valid:

$$
\int_{B}|f(y)| d m_{\nu}(y) \leq 2 m_{\nu}(B) \Phi^{-1}\left(\left(m_{\nu}(B)\right)^{-1}\right)\left\|f \chi_{B}\right\|_{L_{\Phi, \nu}} .
$$

We begin with the boundedness of the maximal operator $M_{\nu}$ on Orlicz spaces $L_{\Phi}\left(\mathbb{R}, d m_{\nu}\right)$.

Theorem 2.2 [5] Let $\Phi$ be a Young function.

(i)The operator $M_{\nu}$ is bounded from $L_{\Phi}\left(\mathbb{R}, d m_{\nu}\right)$ to $W L_{\Phi}\left(\mathbb{R}, d m_{\nu}\right)$, and the inequality

$$
\left\|M_{\nu} f\right\|_{W L_{\Phi, \nu}} \leq C_{0}\|f\|_{L_{\Phi, \nu}}
$$

holds with constant $C_{0}$ independent of $f$.

(ii) The operator $M_{\nu}$ is bounded on $L_{\Phi}\left(\mathbb{R}, d m_{\nu}\right)$, and the inequality

$$
\left\|M_{\nu} f\right\|_{L_{\Phi, \nu}} \leq C_{0}\|f\|_{L_{\Phi, \nu}}
$$

holds with constant $C_{0}$ independent of $f$ if and only if $\Phi \in \nabla_{2}$.

The following theorems were proved in [8].

Theorem 2.3 [8] Let $b \in B M O\left(\mathbb{R}, d m_{\nu}\right)$ and $\Phi \in \mathcal{Y}$. Then the condition $\Phi \in \nabla_{2}$ is necessary and sufficient for the boundedness of $M_{b, \nu}$ on $L_{\Phi}\left(\mathbb{R}, d m_{\nu}\right)$.

Theorem 2.4 [8] Let $\Phi$ be a Young function with $\Phi \in \nabla_{2}$. Then the condition $b \in B M O\left(\mathbb{R}, d m_{\nu}\right)$ is necessary and sufficient for the boundedness of $M_{b, \nu}$ on $L_{\Phi}\left(\mathbb{R}, d m_{\nu}\right)$.

From (2.1) and Theorem 2.4 we deduce the following conclusion.

Corollary 2.1 Let $\Phi$ be a Young function with $\Phi \in \nabla_{2}$. Then the conditions $b^{+} \in B M O\left(\mathbb{R}, d m_{\nu}\right)$ and $b^{-} \in L_{\infty}\left(\mathbb{R}, d m_{\nu}\right)$ are sufficient for the boundedness of $\left[b, M_{\nu}\right]$ on $L_{\Phi}\left(\mathbb{R}, d m_{\nu}\right)$. 
3 Boundedness of fractional maximal operator $M_{\alpha, \nu}$ in the Orlicz spaces $L_{\Phi}\left(\mathbb{R}, d m_{\nu}\right)$

In this section, we shall give a necessary and sufficient condition for the boundedness of $M_{\alpha, \nu}$ on Orlicz spaces $L_{\Phi}\left(\mathbb{R}, d m_{\nu}\right)$ and weak Orlicz spaces $W L_{\Phi}\left(\mathbb{R}, d m_{\nu}\right)$.

In order to prove our main theorem, we also need the following lemma.

Lemma 3.1 If $B_{0}:=B\left(x_{0}, r_{0}\right)$, then $m_{\nu}\left(B_{0}\right)^{\frac{\alpha}{2 \nu+2}} \leq M_{\alpha, \nu} \chi_{B_{0}}(x)$ for every $x \in B_{0}$.

Proof. For $x \in B_{0}$, we get

$$
\begin{aligned}
M_{\alpha, \nu} \chi_{B_{0}}(x) & =\sup _{B \ni x} m_{\nu}\left(B_{0}\right)^{-1+\frac{\alpha}{2 \nu+2}} m_{\nu}\left(B \cap B_{0}\right) \\
& \geq m_{\nu}\left(B_{0}\right)^{-1+\frac{\alpha}{2 \nu+2}} m_{\nu}\left(B_{0} \cap B_{0}\right)=m_{\nu}\left(B_{0}\right)^{\frac{\alpha}{2 \nu+2}} .
\end{aligned}
$$

The following result completely characterizes the boundedness of $M_{\alpha, \nu}$ on Orlicz spaces $L_{\Phi}\left(\mathbb{R}, d m_{\nu}\right)$.

Theorem 3.1 Let $0<\alpha<2 \nu+2, \Phi, \Psi$ be Young functions and $\Phi \in \mathcal{Y}$. The condition

$$
r^{-\frac{\alpha}{2 \nu+2}} \Phi^{-1}(r) \leq C \Psi^{-1}(r)
$$

for all $r>0$, where $C>0$ does not depend on $r$, is necessary and sufficient for the boundedness of $M_{\alpha, \nu}$ from $L_{\Phi}\left(\mathbb{R}, d m_{\nu}\right)$ to $W L_{\Psi}\left(\mathbb{R}, d m_{\nu}\right)$. Moreover, if $\Phi \in \nabla_{2}$, the condition (3.1) is necessary and sufficient for the boundedness of $M_{\alpha, \nu}$ from $L_{\Phi}\left(\mathbb{R}, d m_{\nu}\right)$ to $L_{\Psi}\left(\mathbb{R}, d m_{\nu}\right)$.

Proof. For arbitrary ball $B=B(x, r)$ we represent $f$ as

$$
f=f_{1}+f_{2}, \quad f_{1}(y)=f(y) \chi_{2 B}(y), \quad f_{2}(y)=f(y) \chi_{\mathrm{c}_{(2 B)}}(y), \quad r>0,
$$

and have

$$
M_{\alpha, \nu} f(x)=M_{\alpha, \nu} f_{1}(x)+M_{\alpha, \nu} f_{2}(x) .
$$

Let $y$ be an arbitrary point in $B$. If $B(y, t) \cap{ }^{\complement}(B(x, 2 r)) \neq \emptyset$, then $t>r$. Indeed, if $z \in B(y, t) \cap{ }^{\mathrm{C}}(B(x, 2 r))$, then $t>|y-z| \geq|x-z|-|x-y|>2 r-r=r$.

On the other hand, $B(y, t) \cap^{\mathrm{C}}(B(x, 2 r)) \subset B(x, 2 t)$. Indeed, if $z \in B(y, t) \cap^{\mathrm{C}}(B(x, 2 r))$, then we get $|x-z| \leq|y-z|+|x-y|<t+r<2 t$.

Hence

$$
\begin{aligned}
M_{\alpha, \nu} f_{2}(y) & \lesssim \sup _{t>0} \frac{1}{m_{\nu}(B(y, t))^{1-\frac{\alpha}{2 \nu+2}}} \int_{B(y, t) \cap^{\complement}(B(x, 2 r))}|f(z)| d m_{\nu}(z) \\
& \lesssim \sup _{t>r} \frac{1}{m_{\nu}(B(x, 2 t))^{1-\frac{\alpha}{2 \nu+2}}} \int_{B(x, 2 t)}|f(z)| d m_{\nu}(z) \\
& =\sup _{t>2 r} \frac{1}{m_{\nu}(B(x, t))^{1-\frac{\alpha}{2 \nu+2}}} \int_{B(x, t)}|f(z)| d m_{\nu}(z) \\
& \lesssim \sup _{r<t<\infty} t^{\alpha} \Phi^{-1}\left(m_{\nu}(B(x, t))^{-1}\right)\|f\|_{L_{\Phi}\left(\mathbb{R}, d m_{\nu}\right)(B(x, t))} \\
& \lesssim\|f\|_{L_{\Phi, \nu}} \sup _{r<t<\infty} t^{\alpha} \Phi^{-1}\left(m_{\nu}(B(x, t))^{-1}\right) .
\end{aligned}
$$

Consequently from Hedberg's trick, see [11], and the last inequality, we have

$$
M_{\alpha, \nu} f(y) \lesssim r^{\alpha} M_{\nu} f(y)+\|f\|_{L_{\Phi, \nu}} \sup _{r<t<\infty} t^{\alpha} \Phi^{-1}\left(t^{-2 \nu-2}\right) .
$$


Thus, by (3.1) we obtain

$$
M_{\alpha, \nu} f(x) \lesssim M_{\nu} f(x) \frac{\Psi^{-1}\left(r^{-2 \nu-2}\right)}{\Phi^{-1}\left(r^{-2 \nu-2}\right)}+\|f\|_{L_{\Phi, \nu}} \Psi^{-1}\left(r^{-2 \nu-2}\right) .
$$

Choose $r>0$ so that $\Phi^{-1}\left(r^{-2 \nu-2}\right)=\frac{M_{\nu} f(x)}{C_{0}\|f\|_{L_{\Phi, \nu}}}$. Then

$$
\frac{\Psi^{-1}\left(r^{-2 \nu-2}\right)}{\Phi^{-1}\left(r^{-2 \nu-2}\right)}=\frac{\left(\Psi^{-1} \circ \Phi\right)\left(\frac{M_{\nu} f(x)}{C_{0}\|f\|_{L_{\Phi, \nu}}}\right)}{\frac{M_{\nu} f(x)}{C_{0}\|f\|_{L_{\Phi, \nu}}}} .
$$

Therefore, we get

$$
M_{\alpha, \nu} f(x) \leq C_{1}\|f\|_{L_{\Phi, \nu}}\left(\Psi^{-1} \circ \Phi\right)\left(\frac{M_{\nu} f(x)}{C_{0}\|f\|_{L_{\Phi, \nu}}}\right) .
$$

Let $C_{0}$ be as in (2.4). Then by Theorem 2.2, we have

$$
\begin{aligned}
& \sup _{r>0} \Psi(r) m_{\nu}\left(B, \frac{M_{\alpha, \nu} f(x)}{C_{1}\|f\|_{L_{\Phi, \nu}}}, r\right)=\sup _{r>0} r m_{\nu}\left(B, \Psi\left(\frac{M_{\alpha, \nu} f(x)}{C_{1}\|f\|_{L_{\Phi, \nu}}}\right), r\right) \\
\leq & \sup _{r>0} r m_{\nu}\left(B, \Phi\left(\frac{M_{\nu} f(x)}{C_{0}\|f\|_{L_{\Phi, \nu}}}\right), r\right) \leq \sup _{r>0} \Phi(r) m_{\nu}\left(\frac{M f(x)}{\|M f\|_{W L_{\Phi, \nu}}}, r\right) \leq 1,
\end{aligned}
$$

i.e.

$$
\left\|M_{\alpha, \nu} f\right\|_{W L_{\Psi, \nu}(B)} \lesssim\|f\|_{L_{\Phi, \nu}} .
$$

By taking supremum over $B$ in (3.2), we get

$$
\left\|M_{\alpha, \nu} f\right\|_{W L_{\Psi, \nu}} \lesssim\|f\|_{L_{\Phi, \nu}},
$$

since the constants in (3.2) don't depend on $x$ and $r$.

Let $C_{0}$ be as in (2.5). Since $\Phi \in \nabla_{2}$, by Theorem 2.2, we have

$$
\begin{aligned}
\int_{B} \Psi\left(\frac{M_{\alpha, \nu} f(x)}{C_{1}\|f\|_{L_{\Phi, \nu}}}\right) d m_{\nu}(x) & \leq \int_{B} \Phi\left(\frac{M_{\nu} f(x)}{C_{0}\|f\|_{L_{\Phi, \nu}}}\right) d m_{\nu}(x) \\
& \leq \int_{\mathbb{R}^{n}} \Phi\left(\frac{M f(x)}{\left\|M_{\nu} f\right\|_{L_{\Phi, \nu}}}\right) d x \leq 1,
\end{aligned}
$$

i.e.

$$
\left\|M_{\alpha, \nu} f\right\|_{L_{\Psi, \nu}(B)} \lesssim\|f\|_{L_{\Phi, \nu}} .
$$

By taking supremum over $B$ in (3.3), we get

$$
\left\|M_{\alpha, \nu} f\right\|_{L_{\Psi, \nu}} \lesssim\|f\|_{L_{\Phi, \nu}},
$$

since the constants in (3.3) don't depend on $x$ and $r$.

We shall now prove the necessity. Let $B_{0}=B\left(x_{0}, r_{0}\right)$ and $x \in B_{0}$. By Lemma 3.1, we have $r_{0}^{\alpha} \leq C M_{\alpha, \nu} \chi_{B_{0}}(x)$. Therefore, by Lemma 2.1, we have

$$
\begin{aligned}
r_{0}^{\alpha} & \lesssim \Psi^{-1}\left(m_{\nu}\left(B_{0}\right)^{-1}\right)\left\|M_{\alpha, \nu} \chi_{B_{0}}\right\|_{W L_{\Psi, \nu}\left(B_{0}\right) \lesssim \Psi^{-1}\left(m_{\nu}\left(B_{0}\right)^{-1}\right)\left\|M_{\alpha, \nu} \chi_{B_{0}}\right\|_{W L_{\Psi, \nu}}} \\
& \lesssim \Psi^{-1}\left(m_{\nu}\left(B_{0}\right)^{-1}\right)\left\|\chi_{B_{0}}\right\|_{L_{\Phi, \nu}} \lesssim \frac{\Psi^{-1}\left(r_{0}^{-2 \nu-2}\right)}{\Phi^{-1}\left(r_{0}^{-2 \nu-2}\right)}
\end{aligned}
$$


and

$$
\begin{aligned}
r_{0}^{\alpha} & \lesssim \Psi^{-1}\left(m_{\nu}\left(B_{0}\right)^{-1}\right)\left\|M_{\alpha, \nu} \chi_{B_{0}}\right\|_{L_{\Psi, \nu}\left(B_{0}\right) \lesssim \Psi^{-1}\left(m_{\nu}\left(B_{0}\right)^{-1}\right)\left\|M_{\alpha, \nu} \chi_{B_{0}}\right\|_{L_{\Psi, \nu}}} \\
& \lesssim \Psi^{-1}\left(m_{\nu}\left(B_{0}\right)^{-1}\right)\left\|\chi_{B_{0}}\right\|_{L_{\Phi, \nu}} \lesssim \frac{\Psi^{-1}\left(r_{0}^{-2 \nu-2}\right)}{\Phi^{-1}\left(r_{0}^{-2 \nu-2}\right)}
\end{aligned}
$$

Since this is true for every $r_{0}>0$, we are done.

We recover the following well known result by taking $\Phi(t)=t^{p}$ at Theorem 3.1.

Corollary 3.1 Let $0<\alpha<2 \nu+2$ and $1 \leq p<(2 \nu+2) / \alpha$. Then the condition $1 / q=$ $1 / p-\alpha /(2 \nu+2)$ is necessary and sufficient for the boundedness of $M_{\alpha, \nu}$ from $L_{p}\left(\mathbb{R}, d m_{\nu}\right)$ to $W L_{q}\left(\mathbb{R}, d m_{\nu}\right)$ and for $p>1$ from $L_{p}\left(\mathbb{R}, d m_{\nu}\right)$ to $L_{q}\left(\mathbb{R}, d m_{\nu}\right)$.

\section{Boundedness of fractional maximal commutators $M_{b, \alpha, \nu}$ in the Orlicz spaces $L_{\Phi}\left(\mathbb{R}, d m_{\nu}\right)$}

In this section we investigate the boundedness of the fractional maximal commutator $M_{b, \alpha, \nu}$ and the commutator of the fractional maximal operator, $\left[b, M_{\alpha, \nu}\right]$, in Orlicz spaces $L_{\Phi}\left(\mathbb{R}, d m_{\nu}\right)$.

We recall the definition of the space $B M O\left(\mathbb{R}, d m_{\nu}\right)$.

Definition 4.1 Suppose that $b \in L_{1}^{\text {loc }}\left(\mathbb{R}, d m_{\nu}\right)$, let

$$
\|b\|_{B M O(\nu)}:=\sup _{x \in \mathbb{R}, r>0} \frac{1}{m_{\nu} B(x, r)} \int_{B(x, r)}\left|b(y)-b_{B(x, r)}(x)\right| d m_{\nu}(y),
$$

where

$$
b_{B(x, r)}:=\frac{1}{m_{\nu} B(x, r)} \int_{B(x, r)} b(y) d m_{\nu}(y)
$$

Define

$$
B M O\left(\mathbb{R}, d m_{\nu}\right):=\left\{b \in L_{1}^{\mathrm{loc}}\left(\mathbb{R}, d m_{\nu}\right):\|b\|_{B M O(\nu)}<\infty\right\} .
$$

Modulo constants, the space $B M O\left(\mathbb{R}, d m_{\nu}\right)$ is a Banach space with respect to the norm $\|\cdot\|_{B M O(\nu)}$

We will need the following properties of $B M O$-functions (see [12]):

$$
\|b\|_{B M O(\nu)} \approx \sup _{x \in \mathbb{R}, r>0}\left(\frac{1}{m_{\nu} B(x, r)} \int_{B(x, r)}\left|b(y)-b_{B(x, r)}\right|^{p} d m_{\nu}(y)\right)^{\frac{1}{p}},
$$

where $1 \leq p<\infty$ and the positive equivalence constants are independent of $b$, and

$$
\left|b_{B(x, r)}-b_{B(x, t)}\right| \leq C\|b\|_{B M O(\nu)} \ln \frac{t}{r} \text { for any } 0<2 r<t,
$$

where the positive constant $C$ does not depend on $b, x, r$ and $t$.

Next, we recall the notion of weights. Let $w$ be a locally integrable and positive function on $\left(\mathbb{R}, d m_{\nu}\right)$. The function $w$ is called a Muckenhoupt $A_{1}\left(\mathbb{R}, d m_{\nu}\right)$ weight if there exists a positive constant $C$ such that for any ball $B$

$$
\frac{1}{m_{\nu}(B)} \int_{B} w(x) d m_{\nu}(x) \leq C \underset{x \in B}{\operatorname{ess} \inf } w(x) .
$$


Lemma 4.1 [5, Chapter 1] Let $\omega \in A_{1}\left(\mathbb{R}, d m_{\nu}\right)$, then the reverse Hölder inequality holds, that is, there exist $q>1$ and a positive constant $C$ such that

$$
\left(\frac{1}{m_{\nu}(B)} \int_{B} w(x)^{q} d m_{\nu}(x)\right)^{\frac{1}{q}} \leq \frac{C}{m_{\nu}(B)} \int_{B} w(x) d m_{\nu}(x)
$$

for all balls $B$.

Lemma 4.2 [8] Let $\Phi$ be a Young function with $\Phi \in \Delta_{2}$, B be a ball in $\mathbb{R}$ and $f \in L_{\Phi, \nu}(B)$.

Then we have

$\frac{1}{2 m_{\nu}(B)} \int_{B}|f(x)| d m_{\nu}(x) \leq \Phi^{-1}\left(m_{\nu}(B)^{-1}\right)\|f\|_{L_{\Phi, \nu}} \leq C\left(\frac{1}{m_{\nu}(B)} \int_{B}|f(x)|^{p} d m_{\nu}(x)\right)^{\frac{1}{p}}$

for some $1<p<\infty$, where the positive constant $C$ does not depend on $f$ and $B$.

We have the following result from (4.1) and Lemma 4.2.

Lemma 4.3 Let $b \in B M O\left(\mathbb{R}, d m_{\nu}\right)$ and $\Phi$ be a Young function with $\Phi \in \Delta_{2}$, then

$$
\|b\|_{B M O(\nu)} \approx \sup _{x \in \mathbb{R}, r>0} \Phi^{-1}\left(m_{\nu}\left(B(x, r)^{-1}\right)\left\|b(\cdot)-b_{B(x, r)}\right\|_{L_{\Phi, \nu}(B(x, r))},\right.
$$

where the positive equivalence constants are independent of $b$.

By Theorem 2.2 and Theorem 1.13 in [2] we obtain the following theorem.

Theorem 4.1 [8] Let $b \in B M O\left(\mathbb{R}, d m_{\nu}\right)$ and and $\Phi$ be a Young function. Then the condition $\Phi \in \nabla_{2}$ is necessary and sufficient for the boundedness of $M_{b, \nu}$ on $L_{\Phi}\left(\mathbb{R}, d m_{\nu}\right)$, i.e., the inequality

$$
\left\|M_{b, \nu} f\right\|_{L_{\Phi, \nu}} \leq C_{0}\|b\|_{\nu}\|f\|_{L_{\Phi, \nu}}
$$

holds with constant $C_{0}$ independent of $f$.

The following lemma is the analogue of the Hedberg's trick for the commutator of fractional integral (see [11]).

Lemma 4.4 If $0<\alpha<2 \nu+2$ and $f, b \in L_{1}^{\text {loc }}\left(\mathbb{R}, d m_{\nu}\right)$, then for all $x \in \mathbb{R}$ and $r>0$ we get

$$
\int_{B(x, r)} \frac{|f(y)|}{|x-y|^{2 \nu+2-\alpha}}|b(x)-b(y)| d m_{\nu}(y) \lesssim r^{\alpha} M_{b, \nu} f(x) .
$$

Proof.

$$
\begin{aligned}
& \int_{B(x, r)} \frac{|f(y)|}{|x-y|^{2 \nu+2-\alpha}}|b(x)-b(y)| d m_{\nu}(y) \\
& =\sum_{j=0}^{\infty} \int_{2^{-j-1} r \leq|x-y|<2^{-j} r} \frac{|f(y)|}{|x-y|^{2 \nu+2-\alpha}}|b(x)-b(y)| d m_{\nu}(y) \\
& \lesssim \sum_{j=0}^{\infty}\left(2^{-j} r\right)^{\alpha}\left(2^{-j} r\right)^{-1} \int_{|x-y|<2^{-j} r}|f(y)||b(x)-b(y)| d m_{\nu}(y) \lesssim r^{\alpha} M_{b, \nu} f(x) .
\end{aligned}
$$

For proving our main results, we need the following estimate.

Lemma 4.5 If $b \in L_{1}^{\mathrm{loc}}\left(\mathbb{R}, d m_{\nu}\right)$ and $B_{0}:=B\left(x_{0}, r_{0}\right)$, then

$$
r_{0}^{\alpha}\left|b(x)-b_{B_{0}}\right| \leq C M_{b, \alpha, \nu} \chi_{B_{0}}(x) \text { for every } x \in B_{0} .
$$


Proof. It is well known that

$$
\mathrm{M}_{b, \nu, \alpha} f(x) \leq 2^{2 \nu+2-\alpha} M_{b, \alpha, \nu} f(x),
$$

where $\mathrm{M}_{b, \nu, \alpha}(f)(x)=\sup _{B \ni x} m_{\nu}(B)^{-2 \nu-2+\alpha} \int_{B}|b(x)-b(y)||f(y)| d m_{\nu}(y)$.

Now let $x \in B_{0}$. By using (4.5), we get

$$
\begin{aligned}
& M_{b, \alpha, \nu} \chi_{B_{0}}(x) \geq C \mathrm{M}_{b, \nu, \alpha} f(x)=C \sup _{B \ni x} m_{\nu}(B)^{-2 \nu-2+\alpha} \int_{B}|b(x)-b(y)| \chi_{B_{0}} d m_{\nu}(y) \\
& =C \sup _{B \ni x} m_{\nu}(B)^{-2 \nu-2+\alpha} \int_{B \cap B_{0}}|b(x)-b(y)| d m_{\nu}(y) \\
& \geq C m_{\nu}\left(B_{0}\right)^{-2 \nu-2+\alpha} \int_{B_{0} \cap B_{0}}|b(x)-b(y)| d m_{\nu}(y) \\
& \geq\left|C m_{\nu}\left(B_{0}\right)^{-2 \nu-2+\alpha} \int_{B_{0}}(b(x)-b(y)) d m_{\nu}(y)\right|=C r_{0}^{\alpha}\left|b(x)-b_{B_{0}}\right| .
\end{aligned}
$$

The following theorem gives necessary and sufficient conditions for the boundedness of the operator $M_{b, \alpha, \nu}$ from $L_{\Phi}\left(\mathbb{R}, d m_{\nu}\right)$ to $L_{\Psi}\left(\mathbb{R}, d m_{\nu}\right)$.

Theorem 4.2 Let $0<\alpha<2 \nu+2, b \in B M O\left(\mathbb{R}, d m_{\nu}\right)$ and $\Phi, \Psi$ be Young functions and $\Phi \in \mathcal{Y}$.

1. If $\Phi \in \nabla_{2}$ and $\Psi \in \Delta_{2}$, then the condition

$$
r^{\alpha} \Phi^{-1}\left(r^{-2 \nu-2}\right)+\sup _{r<t<\infty}\left(1+\ln \frac{t}{r}\right) \Phi^{-1}\left(t^{-2 \nu-2}\right) t^{\alpha} \leq C \Psi^{-1}\left(r^{-2 \nu-2}\right)
$$

for all $r>0$, where $C>0$ does not depend on $r$, is sufficient for the boundedness of $M_{b, \alpha, \nu}$ from $L_{\Phi}\left(\mathbb{R}, d m_{\nu}\right)$ to $L_{\Psi}\left(\mathbb{R}, d m_{\nu}\right)$.

2. If $\Psi \in \Delta_{2}$, then the condition (3.1) is necessary for the boundedness of $M_{b, \alpha, \nu}$ from $L_{\Phi}\left(\mathbb{R}, d m_{\nu}\right)$ to $L_{\Psi}\left(\mathbb{R}, d m_{\nu}\right)$.

3. Let $\Phi \in \nabla_{2}$ and $\Psi \in \Delta_{2}$. If the condition

$$
\sup _{r<t<\infty}\left(1+\ln \frac{t}{r}\right) \Phi^{-1}\left(t^{-2 \nu-2}\right) t^{\alpha} \leq C r^{\alpha} \Phi^{-1}\left(r^{-2 \nu-2}\right)
$$

holds for all $r>0$, where $C>0$ does not depend on $r$, then the condition (3.1) is necessary and sufficient for the boundedness of $M_{b, \alpha, \nu}$ from $L_{\Phi}\left(\mathbb{R}, d m_{\nu}\right)$ to $L_{\Psi}\left(\mathbb{R}, d m_{\nu}\right)$.

Proof. 1. For arbitrary $x_{0} \in \mathbb{R}$, set $B=B\left(x_{0}, r\right)$ for the ball centered at $x_{0}$ and of radius $r$. Write $f=f_{1}+f_{2}$ with $f_{1}=f \chi_{2 B}$ and $f_{2}=f \chi_{\mathrm{C}_{(2 B)}}$.

Let $x$ be an arbitrary point in $B$. If $B(x, t) \cap\left\{{ }^{\mathrm{C}}(2 B)\right\} \neq \emptyset$, then $t>r$. Indeed, if $y \in B(x, t) \cap\left\{{ }^{\mathrm{C}}(2 B)\right\}$, then $t>|x-y| \geq\left|x_{0}-y\right|-\left|x_{0}-x\right|>2 r-r=r$.

On the other hand, $B(x, t) \cap\left\{{ }^{\mathrm{C}}(2 B)\right\} \subset B\left(x_{0}, 2 t\right)$. Indeed, if $y \in B(x, t) \cap\left\{{ }^{\mathrm{C}}(2 B)\right\}$, then we get $\left|x_{0}-y\right| \leq|x-y|+\left|x_{0}-x\right|<t+r<2 t$.

Hence

$$
\begin{aligned}
M_{b, \alpha, \nu}\left(f_{2}\right)(x) & =\sup _{t>0} \frac{1}{m_{\nu}\left(B(x, t)^{1-\frac{\alpha}{2 \nu+2}}\right.} \int_{B(x, t) \cap^{\complement}(2 B)}|b(y)-b(x)||f(y)| d m_{\nu}(y) \\
& \leq 2^{n-\alpha} \sup _{t>r} \frac{1}{m_{\nu}\left(B\left(x_{0}, 2 t\right)\right)^{1-\frac{\alpha}{2 \nu+2}}} \int_{B\left(x_{0}, 2 t\right)}|b(y)-b(x)||f(y)| d m_{\nu}(y) \\
& =2^{n-\alpha} \sup _{t>2 r} \frac{1}{m_{\nu}\left(B\left(x_{0}, t\right)\right)^{1-\frac{\alpha}{2 \nu+2}}} \int_{B\left(x_{0}, t\right)}|b(y)-b(x)||f(y)| d m_{\nu}(y) .
\end{aligned}
$$


Therefore, for all $x \in B$ we have

$$
\begin{aligned}
& M_{b, \alpha, \nu}\left(f_{2}\right)(x) \lesssim \sup _{t>2 r} t^{\alpha-2-2 \nu} \int_{B\left(x_{0}, t\right)}|b(y)-b(x)||f(y)| d m_{\nu}(y) \\
& \lesssim \sup _{t>2 r} t^{\alpha-2-2 \nu} \int_{B\left(x_{0}, t\right)}\left|b(y)-b_{B\left(x_{0}, t\right)}\right||f(y)| d m_{\nu}(y) \\
& +\sup _{t>2 r} t^{\alpha-2-2 \nu} \int_{B\left(x_{0}, t\right)}\left|b_{B\left(x_{0}, t\right)}-b_{B}\right||f(y)| d m_{\nu}(y) \\
& +\sup _{t>2 r} t^{\alpha-2-2 \nu} \int_{B\left(x_{0}, t\right)}\left|b_{B}-b(x)\right||f(y)| d m_{\nu}(y)=J_{1}+J_{2}+J_{3} .
\end{aligned}
$$

Applying Hölder's inequality, by (2.2), (4.2), (4.3) and (2.2) we get

$$
\begin{aligned}
J_{1}+J_{2} \lesssim & \sup _{t>2 r} t^{\alpha-2-2 \nu} \int_{B\left(x_{0}, t\right)}\left|b(y)-b_{B\left(x_{0}, t\right)}\right||f(y)| d m_{\nu}(y) \\
& +\sup _{t>2 r} t^{\alpha-2-2 \nu}\left|b_{B\left(x_{0}, r\right)}-b_{B\left(x_{0}, t\right)}\right| \int_{B\left(x_{0}, t\right)}|f(y)| d m_{\nu}(y) \\
\lesssim & \sup _{t>2 r} t^{\alpha-2-2 \nu}\left\|b(\cdot)-b_{B\left(x_{0}, t\right)}\right\|_{L_{\widetilde{\Phi}}\left(B\left(x_{0}, t\right)\right)}\|f\|_{L_{\Phi, \nu}\left(B\left(x_{0}, t\right)\right)} \\
& +\sup _{t>2 r} t^{\alpha-2-2 \nu}\left|b_{B\left(x_{0}, r\right)}-b_{B\left(x_{0}, t\right)}\right| t^{n} \Phi^{-1}\left(m_{\nu}\left(B\left(x_{0}, t\right)\right)^{-1}\right)\|f\|_{L_{\Phi, \nu}\left(B\left(x_{0}, t\right)\right)} \\
\lesssim & \|b\|_{\nu} \sup _{t>2 r} \Phi^{-1}\left(m_{\nu}\left(B\left(x_{0}, t\right)\right)^{-1}\right) t^{\alpha}\left(1+\ln \frac{t}{r}\right)\|f\|_{L_{\Phi, \nu}\left(B\left(x_{0}, t\right)\right)} \\
\lesssim & \|b\|_{\nu}\|f\|_{L_{\Phi, \nu}} \sup _{t>2 r}\left(1+\ln \frac{t}{r}\right) t^{\alpha} \Phi^{-1}\left(t^{-2 \nu-2}\right) .
\end{aligned}
$$
get

A geometric observation shows $2 B \subset B(x, 3 r)$ for all $x \in B$. Using Lemma 4.4, we

$$
\begin{aligned}
J_{0}(x) & :=M_{b, \alpha, \nu}\left(f_{1}\right)(x) \lesssim\left|b, I_{\alpha}\right|\left(\left|f_{1}\right|\right)(x)=\int_{2 B} \frac{|b(y)-b(x)|}{|x-y|^{n-\alpha}}|f(y)| d m_{\nu}(y) \\
& \lesssim \int_{B(x, 3 r)} \frac{|b(y)-b(x)|}{|x-y|^{n-\alpha}}|f(y)| d m_{\nu}(y) \lesssim r^{\alpha} M_{b, \nu} f(x) .
\end{aligned}
$$

Consequently for all $x \in B$ we get

$$
J_{0}(x)+J_{1}+J_{2} \lesssim\|b\|_{\nu} r^{\alpha} M_{b, \nu} f(x)+\|b\|_{\nu}\|f\|_{L_{\Phi, \nu}} \sup _{t>2 r}\left(1+\ln \frac{t}{r}\right) t^{\alpha} \Phi^{-1}\left(t^{-2 \nu-2}\right) .
$$

Thus, by (4.6) we obtain

$$
J_{0}(x)+J_{1}+J_{2} \lesssim\|b\|_{\nu}\left(M_{b, \nu} f(x) \frac{\Psi^{-1}\left(r^{-2 \nu-2}\right)}{\Phi^{-1}\left(r^{-2 \nu-2}\right)}+\Psi^{-1}\left(r^{-2 \nu-2}\right)\|f\|_{L_{\Phi, \nu}}\right) .
$$

Choose $r>0$ so that $\Phi^{-1}\left(r^{-2 \nu-2}\right)=\frac{M_{b, \nu} f(x)}{C_{0}\|b\|_{\nu}\|f\|_{L_{\Phi, \nu}}}$. Then

$$
\frac{\Psi^{-1}\left(r^{-2 \nu-2}\right)}{\Phi^{-1}\left(r^{-2 \nu-2}\right)}=\frac{\left(\Psi^{-1} \circ \Phi\right)\left(\frac{M_{b, \nu} f(x)}{C_{0}\|b\|_{\nu}\|f\|_{L_{\Phi, \nu}}}\right)}{\frac{M_{b, \nu} f(x)}{C_{0}\|b\|_{\nu}\|f\|_{L_{\Phi, \nu}}}} .
$$


Therefore, we get

$$
J_{0}(x)+J_{1}+J_{2} \leq C_{1}\|b\|_{\nu}\|f\|_{L_{\Phi, \nu}}\left(\Psi^{-1} \circ \Phi\right)\left(\frac{M_{b, \nu} f(x)}{C_{0}\|b\|_{\nu}\|f\|_{L_{\Phi, \nu}}}\right) .
$$

Let $C_{0}$ be as in (4.4). Consequently by Theorem 4.1 and (2.3) we have

$$
\begin{aligned}
\int_{B} \Psi\left(\frac{J_{0}(x)+J_{1}+J_{2}}{C_{1}\|b\|_{\nu}\|f\|_{L_{\Phi, \nu}}}\right) d m_{\nu}(x) & \leq \int_{B} \Phi\left(\frac{M_{b, \nu} f(x)}{C_{0}\|b\|_{\nu}\|f\|_{L_{\Phi, \nu}}}\right) d m_{\nu}(x) \\
& \leq \int_{\mathbb{R}^{n}} \Phi\left(\frac{M_{b, \nu} f(x)}{\left\|M_{b, \nu} f\right\|_{L_{\Phi, \nu}}}\right) d m_{\nu}(x) \leq 1,
\end{aligned}
$$

i.e.

$$
\left\|J_{0}(\cdot)+J_{1}+J_{2}\right\|_{L_{\Psi, \nu}(B)} \lesssim\|b\|_{\nu}\|f\|_{L_{\Phi, \nu}} .
$$

By (4.3), (2.2) and condition (4.6), we also get

$$
\begin{aligned}
\left\|J_{3}\right\|_{L_{\Psi, \nu}(B)} & =\left\|\operatorname { s u p } _ { t > 2 r } \frac { 1 } { m _ { \nu } ( B ( x _ { 0 } , t ) ) ^ { 1 - \frac { \alpha } { 2 \nu + 2 } } } \int _ { B ( x _ { 0 } , t ) } \left|b(\cdot)-b_{B}\left\|f(y) \mid d m_{\nu}(y)\right\|_{L_{\Psi, \nu}(B)}\right.\right. \\
& \approx\left\|b(\cdot)-b_{B}\right\|_{L_{\Psi, \nu}(B)} \sup _{t>2 r} t^{\alpha-2-2 \nu} \int_{B\left(x_{0}, t\right)}|f(y)| d m_{\nu}(y) \\
& \lesssim\|b\|_{\nu} \frac{1}{\Psi^{-1}\left(m_{\nu}(B)^{-1}\right)} \sup _{t>2 r} \Phi^{-1}\left(m_{\nu}\left(B\left(x_{0}, t\right)\right)^{-1}\right) t^{\alpha}\|f\|_{L_{\Phi, \nu}\left(B\left(x_{0}, t\right)\right)} \\
& \lesssim\|b\|_{\nu} \frac{1}{\Psi^{-1}\left(m_{\nu}(B)^{-1}\right)}\|f\|_{L_{\Phi, \nu}} \sup _{t>2 r} t^{\alpha} \Phi^{-1}\left(m_{\nu}\left(B\left(x_{0}, t\right)\right)^{-1}\right) \lesssim\|b\|_{\nu}\|f\|_{L_{\Phi, \nu}} .
\end{aligned}
$$

Consequently, we have

$$
\left\|J_{3}\right\|_{L_{\Psi, \nu}(B)} \lesssim\|b\|_{\nu}\|f\|_{L_{\Phi, \nu}} .
$$

Combining (4.8) and (4.9), we get

$$
\left\|M_{b, \alpha, \nu} f\right\|_{L_{\Psi, \nu}(B)} \lesssim\|b\|_{\nu}\|f\|_{L_{\Phi, \nu}} .
$$

By taking supremum over $B$ in (4.10), we get

$$
\left\|M_{b, \alpha, \nu} f\right\|_{L_{\Psi, \nu}} \lesssim\|b\|_{\nu}\|f\|_{L_{\Phi, \nu}},
$$

since the constants in (4.10) don't depend on $x_{0}$ and $r$.

2. We shall now prove the second part. Let $B_{0}=B\left(x_{0}, r_{0}\right)$ and $x \in B_{0}$. By Lemma 4.5, we have $r_{0}^{\alpha}\left|b(x)-b_{B_{0}}\right| \leq C M_{b, \alpha, \nu} \chi_{B_{0}}(x)$. Therefore, by Lemmas 4.3 and 2.1

$$
\begin{aligned}
r_{0}^{\alpha} & \lesssim \frac{\left\|M_{b, \alpha, \nu} \chi_{B_{0}}\right\|_{L_{\Psi, \nu}\left(B_{0}\right)}}{\left\|b(\cdot)-b_{B_{0}}\right\|_{L_{\Psi, \nu}\left(B_{0}\right)}} \lesssim \Psi^{-1}\left(m_{\nu}\left(B_{0}\right)^{-1}\right)\left\|M_{b, \alpha, \nu} \chi_{B_{0}}\right\|_{L_{\Psi, \nu}\left(B_{0}\right)} \\
& \lesssim \Psi^{-1}\left(m_{\nu}\left(B_{0}\right)^{-1}\right)\left\|M_{b, \alpha, \nu} \chi_{B_{0}}\right\|_{L_{\Psi, \nu}} \lesssim \Psi^{-1}\left(m_{\nu}\left(B_{0}\right)^{-1}\right)\left\|\chi_{B_{0}}\right\|_{L_{\Phi, \nu}} \lesssim \frac{\Psi^{-1}\left(r_{0}^{-2 \nu-2}\right)}{\Phi^{-1}\left(r_{0}^{-2 \nu-2}\right)} .
\end{aligned}
$$

Since this is true for every $r_{0}>0$, we are done.

3 . The third statement of the theorem follows from the first and second parts of the theorem.

If we take $\Phi(t)=t^{p}$ and $\Psi(t)=t^{q}$ in Theorem 4.2 we get the following corollary. 
Corollary 4.1 Let $1<p<\infty, 0<\alpha<(2 \nu+2) / p$ and $b \in B M O\left(\mathbb{R}, d m_{\nu}\right)$. Then $M_{b, \alpha, \nu}$ is bounded from $L_{p}\left(\mathbb{R}, d m_{\nu}\right)$ to $L_{q}\left(\mathbb{R}, d m_{\nu}\right)$ if and only if $\frac{1}{q}=\frac{1}{p}-\frac{\alpha}{2 \nu+2}$.

By (2.1) and Theorems 4.2 and 3.1 we get the following corollary.

Corollary 4.2 Let $0<\alpha<2 \nu+2, b \in B M O\left(\mathbb{R}, d m_{\nu}\right), b^{-} \in L_{\infty}\left(\mathbb{R}, d m_{\nu}\right)$ and $\Phi, \Psi$ be Young functions with $\Phi \in \nabla_{2} \cap \mathcal{Y}$ and $\Psi \in \Delta_{2}$. Let also the condition (4.6) is satisfied. Then the operator $\left[b, M_{\alpha, \nu}\right]$ is bounded from $L_{\Phi}\left(\mathbb{R}, d m_{\nu}\right)$ to $L_{\Psi}\left(\mathbb{R}, d m_{\nu}\right)$.

The following theorem is valid.

Theorem 4.3 Let $0<\alpha<2 \nu+2, b \in L_{1}^{\text {loc }}\left(\mathbb{R}, d m_{\nu}\right)$ and $\Phi, \Psi$ be Young functions with $\Phi \in \mathcal{Y}$.

1. If $\Phi \in \nabla_{2}, \Psi \in \Delta_{2}$ and the condition (4.6) holds, then the condition $b \in B M O\left(\mathbb{R}, d m_{\nu}\right)$ is sufficient for the boundedness of $M_{b, \alpha, \nu}$ from $L_{\Phi}\left(\mathbb{R}, d m_{\nu}\right)$ to $L_{\Psi}\left(\mathbb{R}, d m_{\nu}\right)$.

2. If $\Psi^{-1}(t) \lesssim \Phi^{-1}(t) t^{-\alpha /(2 \nu+2)}$, then the condition $b \in B M O\left(\mathbb{R}, d m_{\nu}\right)$ is necessary for the boundedness of $M_{b, \alpha, \nu}$ from $L_{\Phi}\left(\mathbb{R}, d m_{\nu}\right)$ to $L_{\Psi}\left(\mathbb{R}, d m_{\nu}\right)$.

3. If $\Phi \in \nabla_{2}, \Psi \in \Delta_{2}, \Psi^{-1}(t) \approx \Phi^{-1}(t) t^{-\alpha /(2 \nu+2)}$ and the condition (4.7) holds, then the condition $b \in B M O\left(\mathbb{R}, d m_{\nu}\right)$ is necessary and sufficient for the boundedness of $M_{b, \alpha, \nu}$ from $L_{\Phi}\left(\mathbb{R}, d m_{\nu}\right)$ to $L_{\Psi}\left(\mathbb{R}, d m_{\nu}\right)$.

Proof. 1. The first statement of the theorem follows from the first part of the Theorem 4.2.

2. We shall now prove the second part. Suppose that $M_{b, \alpha, \nu}$ is bounded from $L_{\Phi}\left(\mathbb{R}, d m_{\nu}\right)$ to $L_{\Psi}\left(\mathbb{R}, d m_{\nu}\right)$. Choose any ball $B=B(x, r)$ in $\mathbb{R}$, by $(2.2)$

$$
\begin{aligned}
& \frac{1}{m_{\nu}(B)} \int_{B}\left|b(y)-b_{B}\right| d m_{\nu}(y)=\frac{1}{m_{\nu}(B)} \int_{B}\left|\frac{1}{m_{\nu}(B)} \int_{B}(b(y)-b(z)) d m_{\nu}(z)\right| d m_{\nu}(y) \\
& \leq \frac{1}{m_{\nu}(B)^{2}} \int_{B} \int_{B}|b(y)-b(z)| d m_{\nu}(z) d m_{\nu}(y) \\
& =\frac{1}{m_{\nu}(B)^{1+\frac{\alpha}{2 \nu+2}}} \int_{B} \frac{1}{m_{\nu}(B)^{1-\frac{\alpha}{2 \nu+2}}} \int_{B}|b(y)-b(z)| \chi_{B}(z) d m_{\nu}(z) d m_{\nu}(y) \\
& \leq \frac{1}{m_{\nu}(B)^{1+\frac{\alpha}{2 \nu+2}}} \int_{B} M_{b, \alpha, \nu}\left(\chi_{B}\right)(y) d m_{\nu}(y) \\
& \leq \frac{2}{m_{\nu}(B)^{1+\frac{\alpha}{2 \nu+2}}}\left\|M_{b, \alpha, \nu}\left(\chi_{B}\right)\right\|_{L_{\Psi}(B)}\|1\|_{L_{\widetilde{\Psi}}(B)} \leq \frac{C}{m_{\nu}(B)^{\frac{\alpha}{2 \nu+2}}} \frac{\Psi^{-1}\left(m_{\nu}(B)^{-1}\right)}{\Phi^{-1}\left(m_{\nu}(B)^{-1}\right)} \leq C .
\end{aligned}
$$

Thus $b \in B M O\left(\mathbb{R}, d m_{\nu}\right)$.

3. The third statement of the theorem follows from the first and second parts of the theorem.

If we take $\Phi(t)=t^{p}$ and $\Psi(t)=t^{q}$ in Theorem 4.3 we get the following corollary.

Corollary 4.3 Let $1<p<\infty, 0<\alpha<(2 \nu+2) / p$ and $\frac{1}{q}=\frac{1}{p}-\frac{\alpha}{2 \nu+2}$. Then $M_{b, \alpha, \nu}$ is bounded from $L_{p}\left(\mathbb{R}, d m_{\nu}\right)$ to $L_{q}\left(\mathbb{R}, d m_{\nu}\right)$ if and only if $b \in B M O\left(\mathbb{R}, d m_{\nu}\right)$.

Acknowledgements The authors thank the referee(s) for careful reading the paper and useful comments. The research of Z. Safarov was partially supported by the Grant of 1st Azerbaijan-Russia Joint Grant Competition (Agreement Number No. EIF-BGM-4-RFTF1/ 2017-21/01/1-M-08). 


\section{References}

1. Abdelkefi, C., Sifi, M.: Dunkl translation and uncentered maximal operator on the real line, JIPAM. J. Inequal. Pure Appl. Math. 8(3), Article 73, 11 pp. (2007).

2. Agcayazi, M., Gogatishvili, A., Koca, K., Mustafayev, R.: A note on maximal commutators and commutators of maximal functions, J. Math. Soc. Japan, 67(2), 581-593 (2015).

3. Coifman, R.R., Rochberg, R., Weiss, G.: Factorization theorems for Hardy spaces in several variables, Ann. of Math., 103 (3), 611-635 (1976).

4. Cianchi, A.: Strong and weak type inequalities for some classical operators in Orlicz spaces, J. London Math. Soc., 60 (1), 247-286 (1999).

5. Genebashvili, I., Gogatishvili, A., Kokilashvili, V., Krbec, M.: Weight theory for integral transforms on spaces of homogeneous type, Longman, Harlow, (1998).

6. Guliyev, V.S., Mammadov, Y.Y.: Some estimations for Riesz potentials in terms maximal and fractional maximal functions associated with the Dunkl operator on the real line, Trans. Natl. Acad. Sci. Azerb. Ser. Phys.-Tech. Math. Sci. 27 (7), Math. Mech., 71-76 (2007).

7. Guliyev, V.S., Mammadov, Y.Y. On fractional maximal function and fractional integral associated with the Dunkl operator on the real line, J. Math. Anal. Appl., 353 (1), 449-459 (2009).

8. Guliyev, V.S., Mammadov, Y.Y., Muslumova, F.A.: Boundedness characterization of maximal commutators on Orlicz spaces in the Dunkl setting, Journal of Mathematical Study, 53 (1), 1-21 (2019).

9. Dunkl, C.F.: Differential-difference operators associated with reflections groups, Trans. Amer. Math. Soc., 311, 167-183 (1989).

10. Dunkl, C.F., Xu, Y.: Orthogonal Polynomials of Several Variables, 2nd edn, Encyclopedia of Mathematics and its Applications, 155 (Cambridge University Press, Cambridge, 2014).

11. Hedberg, L.I.: On certain convolution inequalities, Proc. Amer. Math. Soc., 36, 505510 (1972).

12. Janson, S.: Mean oscillation and commutators of singular integral operators, Ark. Mat., 16, 263-270 (1978).

13. Kamoun, L.: Besov-type spaces for the Dunkl operator on the real line, J. Comput. Appl. Math., 199, 56-67 (2007).

14. Kita, H.: On maximal functions in Orlicz spaces, Proc. Amer. Math. Soc., 124, 30193025 (1996).

15. Mammadov, Y.Y.: Some embeddings into the modified Morrey spaces associated with the Dunkl operator on the real line, Trans. Natl. Acad. Sci. Azerb. Ser. Phys.-Tech. Math. Sci. 29 (1), Math. Mech., 111-120 (2009).

16. Mejjaoli, H., Trimeche, Kh.: Harmonic analysis associated with the Dunkl-Bessel Laplace operator and a mean value property, Fract. Calc. Appl. Anal., 4 (4), 443-480 (2001).

17. Mourou, M.A.: Transmutation operators associated with a Dunkl-type differentialdifference operator on the real line and certain of their applications, Integral Transforms Spec. Funct., 12 (1), 77-88 (2001).

18. Orlicz, W.: Über eine gewisse Klasse von Räumen vom Typus B, Bull. Acad. Polon. A, 207-220 (1932); reprinted in: Collected Papers, PWN, Warszawa, 217-230 (1988).

19. Orlicz, W.: Über Räume $\left(L^{M}\right)$, Bull. Acad. Polon. A, 93-107 (1936); reprinted in: Collected Papers, PWN, Warszawa, 345-359 (1988).

20. Rao, M.M., Ren, Z.D.: Theory of Orlicz Spaces, M. Dekker, Inc., New York, (1991).

21. Soltani, F.: $L_{p}$-Fourier multipliers for the Dunkl operator on the real line, J. Funct. Anal., 209, 16-35 (2004). 
22. Soltani, F.: On the Riesz-Dunkl potentials, Proc. Inst. Math. Mech. Natl. Acad. Sci. Azerb. 40 (2), 14-21 (2014).

23. Stein, E.M.: Harmonic Analysis: Real Variable Methods, Orthogonality and Oscillatory Integrals, Princeton Univ. Press, Princeton NJ, (1993).

24. Trimeche, K.: Paley-Wiener theorems for the Dunkl transform and Dunkl translation operators, Integral Transforms Spec. Funct. 13, 17-38 (2002). 